Asplenium trichomanes L., Ceterach officinarum Lam. et DO., Urtica dioica L., r, Parietaria judaica L., Euphorbia Wulfenii Hoppe, Acer monspessulanum L., Rubus ulmifolius Schott, Prunus mahaleb L., Coronilla emeroides. Boiss. et Sprun., Hedera helix L., Rubia peregrina L., Campanula pyramidalis L., Helichrysum italicum (Roth) Guss.

Bis an die hintere Höhlenwand, also bis in eine Tiefe von $7 \mathrm{~m}$ reicht Asplenium trichomanes $\left(\mathrm{L}=\frac{1}{18 \mathrm{z}}\right.$ ). Dann folgt Parietaria judaica bis $6 \mathrm{~m}$, Rubia peregrina bis $5.6 \mathrm{~m}$, Rubus ulmifolius bis $5 \mathrm{~m}$ und Urtica dioica bis $2 \mathrm{~m}\left(\mathrm{~L}=\frac{1}{2 \cdot 64}\right)$. Alle Pflanzen besitzen euphotometrische Blätter.

Von Moosen reicht bis $7 \mathrm{~m}$ Rhynchostegiella algiriana (Brid.) Broth, das hier nur in sterilen Exemplaren gefunden wurde; bei $5 \mathrm{~m}$ Tiefe sammelte ich außerdem Fissidens bryoides Hedw.

Auch hier sind die Gesteinstrümmer am Boden und die Höhlenwände mit dem grünen Überzug des Protococcus viridis Ag. bedeckt, der bis $7 \mathrm{~m}$ Tiefe reicht. Daneben sammelte ich hier an der hinteren Höhlenwand $\left(L=\frac{1}{187}\right)$ auch Schizothrix calcicola (Ag.) Gom.

Die Temperatur betrug am Höhleneingang im Sehatten (17. VIII., 9 Uhr vorm.) $+17 \cdot 2^{\circ} \mathrm{C}$, in $1 \mathrm{~m}$ Tiefe $16 \cdot 3^{\circ} \mathrm{C}$, in $3 \mathrm{~m}$ Tiefe $15 \cdot 6^{\circ} \mathrm{C}$, in $7 \mathrm{~m}$ Tiefe nur mehr $14 \cdot 9^{\circ} \mathrm{O}$. Die Differenz zwischen der Eingangstemperatur und jener bei $7 \mathrm{~m}$ Tiefe betrug also $2 \cdot 3^{\circ} \mathrm{C}$.

Es ist mir noch eine angenehme Pflicht, am Schlusse allen jenen Herren zu danken, die diese Arbeit durch ihre Mithilfe unterstützten. Herr Gymnasialdirektor Julius Glowacki in Graz hatte die Freundlichkeit, die Moose zu bestimmen, während Herr Dr. Ernst Lemmermann in Bremen die Bestimmung der Algen durchführte. Herrn Professor Alfons Paulin in Laibach verdanke ich die Revision der Farnpflanzen. Schließlich bin ich noch Herrn Hofrat Dr. Julius R. v. Wiesner zu großem Danke verpflichtet, der der Arbeit ein großes Interesse entgegenbrachte und sie durch manche Ratschläge förderte.

\title{
Keimungsphysiologische Untersuchungen.
}

Von Edmond Heilpern (Wièn).

Aus dem pflanzenphysiologischen Institut der k. k. Universität in Wien, Nr. 69 der zweiten Folge.

(Mit 2 Textabbildungen.)

\section{Fragestellung.}

Es existieren in der botanischen und gärtnerischen Literatur viele Angaben darüber, daß die Kälte, bzw. der Frost auf die Samenkeimung fördernd und beschleunigend einwirken ${ }^{1}$ ). In dieser Arbeit wurde der

1) G ü m b el H., Untersuchungen über die Keimungsverhältnisse verschiedener Unkräuter. Disssert. Merseburg, 1912, p. 28 ff.

Kinzel W., Über die Wirkung des Durchfrierens der Samen auf die Keimung. Ber. d. deutseh. bot. Ges., 1908, p. 642 .

Kinzel W., Frost und Licht als beeinflussende Faktoren bei der Samenkeimung. Stuttgart, 1913. Verlag von Eugen Ulmer. Tabellen. 
Versuch gemacht, das Problem exakter zu fassen. Der Begriff „Frost und Kälte" ist ein ziemlich unbestimmter. Ich steilte deshalb die "Frage folgendermaßen :

Hat die Temperatur von $0^{0}$ die Fähigkeit, die Keimung der Samen in bestimmter Weise zu beeinflussen? Ist die Beinflussung verschieden, je nachdem die Samen in Wasser, Luft, Eis oder. Schnee von dieser Temperatur lagen?

Wie das nun bei wissenschaftlichen Untersuchungen geht, erstreckte sich meine Arbeit bald auch auf andere Gebiete, wie: auf den Einfluß von Licht und Dunkel, auf die mögliche Abkürzung der Ruheperiode, auf die Keimung dimorpher Samen u. a. m. Das begründet auch den Titel, den ich meiner Arbeit gegeben habe, denn es sind mehr oder minder lose zusammenbängende Ergebnisse keimungsphysiologischer Untersuchungen.

\section{Versuchsanstellung.}

Da ich zunächst den Einflu@ von $0^{0}$ auf Samen zu untersuchen beabsichtigte, wurden die Versuche in folgender Weise angeordnet.

Die trocken in einem Zimmer von gewöhnlicher Temperatur (kein Laboratorium) aufbewahrten Samen, wurden in offenen Gläschen, in welchen sich das betreffende Substrat (Schnee, Eis, Wasser) befand, und zwar darin eingepackt oder darauf liegend in den Kühlraum gestellt. In diesem herrschte eine Temperatur von zirka $0^{\circ}$, doch kamen im Laufe des Tages Schwankungen der Temperatur vor. Diese verliefen im Winter so, daß in der Nacht die Temperatur auf ein weniges unter $0^{0}$ sank (höchstens $-3^{0}$ ) und gegen Mittag etwas über $0^{0}$ stieg $^{1}$ ). Nachdem die Samen eine bestimmte Zeit ( 8 Tage bis 6 Wochen) im KübIraum gestanden hatten, wurden sie im Versuchsraum des Glashauses des Wiener pflanzenphysiologischen Institutes zum Keimen ausgelegt, und zwar in Petrischalen auf Filtrierpapier. Gleichzeitig wurde der entsprechende Parallelversuch mit den lufttrocken, bei Zimmertemperatur aufbewabrten Samen aufgestellt. Es wurde stets eine Reihe im Licht und eine im Dunkel zur Keimung ausgelegt. Zur Herstellung eines dunklen Raumes wurden Blechstürze über die Petrischalen gegeben. Alle zahlenmäßig angeführten Resultate sind das Mittel aus mebreren Versuchen.

\section{Einfluß der Temperatur von $0^{\circ}$ auf Samen ohne Ruheperiode.}

Zufolge vieler Literaturangaben soll die Kälte ganz allgemein fördernd auf die Keimung wirken. Das ist nun, wie meine Versuche gezeigt haben, für die Temperaturen $0^{\circ}$ und um $0^{\circ}$ bei den von mir untersuchten Samen nicht richtig.

Zur Untersuchung kamen käufliche Samen, bzw. Früchtchen von: Avena sativa, Adonis vernalis, Bastardklee, Carduus acanthoides, Centaurea cyanus, Esche, Hainbuche, Winterhafer, Helianthus annuus, Hor-

1) Es sei mir gestattet an dieser Stelle der Direktion der Kühl- und Gefrierhaus A. G., Wien II., meinen herzlichsten Dank auszuspreehen, denn ihr außerordentlich liebenswürdiges Entgegenkommen ermöglichte es mir, in den ausgezeichnet gekühlten Räumen dieser Gesellschaft meine Versuche aufzustellen. 
deum vulgare, Lolium perenne, Panicum miliaceum, Physalis Francheti, Winterraps, Robinia pseudacacia, Winterroggen, Rotklee, Sommerrübsen, Secale cereale, Sinapis arvensis, Triticum aestivum, Winterweizen, Winterwicke und Zea mays.

Dabei war übereinstimmend zu beobachten, daß̧ die Kälte auf die Keimung in keiner Weise einen Einfluß hat. In keinem Fall zeigte sich eine Beschleunigung der Keimung oder eine Erhöhung des Keimprozentes.

Nur bei Robinia wurde das Keimprozent herabgesetzt, je länger man die Samen der Kälte aussetzte.

Während des Aufenthaltes in Eis, Schnee usw. fand mit einer Ausnahme nie ein Auskeimen statt. Dieses war immer erst im Institut bei günstiger Temperatur, auf Filtrierpapier ausgelegt, zu beobachten.

Eine Besonderheit stellt jedenfalls Centaurea cyanus dar, denn hier keimten im Eis, Schnee usw. nicht weniger als 70\% Samen innerhalb 15 Tagen aus. Der Centaurea-Same hat also die Fähigkeit, bei tiefen Temperaturen zu keimen.

Bei dieser Gelegenheit möchte ich an eine andere interessante Eigentümlichkeit erinnern. Centaurea cyanus gedeiht nur in Kornfeldern, ist aber im angrenzenden Boden höchstens ausnahmsweise anzutreffen, obgleich Boden-, Feuchtigkeitsverhältnisse usw. ganz ähnlich sind.

\section{Zur Ruheperiode von Samen.}

Sehen wir uns die bisher verwendeten Samen an, so ist eine Anzahl von ihnen (Avena usw.) in der Lage, jederzeit auszukeimen. Von den anderen ist nicht bekannt, ob sie das, was man eine Rubeperiode nennen könnte, besitzen. Nun lag der Gedanke nabe - besonders nach den Untersuchungen von Kinzel - daß die Temperatur von $0^{\circ} \mathbf{a b}$. kürzend auf eine vorhandene Ruheperiode einwirken würde. Ich war also vor die Notwendigkeit gestellt, nach Samen mit Ruheperiode zu suchen; nach den schon vorhandenen Angaben ${ }^{1}$ ) besitzen eine solche die Gattungen Amarantus, Fraxinus, Carpinus, Genista, Digitalis, Betula und Sisymbrium, welche sich für meine Versuche als nicht geeignet erwiesen. Es gelang mir, eine Ruheperiode bei: Acer platanoides, Aethusa cynapium, Geranium pyrenaicum, Oenothera biennis, Ranunculus acer und Silene acaulis festzustellen, worauf weiter unten noch näher eingegangen wird.

Für die Untersuchungen über den Einfluß der Temperaturen von $0^{0}$ erwiesen auch sie sich nicht brauchbar, insoferne man nämlich auf ein $\mathrm{e}$ Abkürzung der Ruheperiode hoffte. Es hat sich gezeigt, daß, ganz gleichgültig, ob die Samen lange oder kurze Zeit in der Kälte, in Eis, Schnee, Wasser oder Luft gehalten wurden, ein Unterschied in der Auskeimung gegenüber den Kontrollexemplaren niemals zu bemerken war.

1) B a a r H., Über den Einfluß des Lichtes auf die Samenkeimung und seine Abhängigkeit von anderen Faktoren. Sitzungsber. d. kaiserl. Akad. d. Wiss. in Wien, mathem.-naturw. Klasse, Bd. CXXI, Abt. I, Juli 1912, p. 35.

Kienitz M., Über Ausführung von Keimproben. Bot. Centralblatt, 1880, I., p. 52.

No bb e F., Handbuch der Samenkunde. Berlin, 1876, pp. 352, 361. 
1. Zur Keimung von Samen verschiedener Provenienz und verschiedenen Erntedatums.

Um Samen mit Ruheperiode zu finden, dachte ich zuerst an Alperpflanzen. Leider war ich aber nicht in der Lage, mir solche Samen in ausreichender Menge zu verschaffen, so das sich meine Untersuchungen hauptsächlich auf die Samen nicht-alpiner Pflanzen erstreckten.

Es lag auch die Annahme nahe, daß durch systematisches Auslegen von zu verschiedenen Jahreszeiten und an verschiedenen Orten gesammelten Samen derselben Art Unterschiede im Keimprozent auftreten müßten.

Untersucht wurden nachfolgende selbst gesammelte $\left.{ }^{1}\right)$ Samen:

Acer platanoides (1), Alliaria officinalis (2), Aethusa cynapium (1), Gentiana pannonica (1), Geranium pyrenaicum (3), Leontodon danubialis (8), Melandrium album (2), Oenothera biennis (1), Plantago lanceolata (2), Ranunculus acer (2), Sambucus nigra (1), Silene acaulis (2), Sinapis arvensis (2), Tragopogon dubius (4) und Ulmus campestris (2). Es sind dies also 15 verschiedene Arten. Die neben dem Namen stehende Zahl zeigt an, von wie viel verschiedenen Standorten, bzw. Erntedaten die Samen stammen.

Bei all diesen Samen zeigt sich durchwegs die Erscheinung, daßs die Samen - entgegen der Meinung vieler Forscher ganz gleich auskeimen, daß also Provenienz und Erntedatum bei der Keimung der von mir untersuchten Samen keine Rolle spielen.

Besonders deutlich trat mir dies bei Leontodon entgegen, welchen ich zu den versehiedensten Zeiten und an den verschiedensten Orten erntete, so aus der Umgebung von Wien, am Semmering (1000 m) und am Schneeberg $(1600 \mathrm{~m})$, also in Gegenden, die in bezug auf Bodenbeschaffenheit und meteorologische Verbältnisse die größten Unterschiede aufweisen. Ferner sammelte ich die Samen von Mai bis September, also zu ganz verschiedenen Jahreszeiten. Alle aber keimten in 11-15 Tagen zu 85-100\% aus, und zwar im Licht und Dunkel gleich gut.

2. Keimungsverlauf bei Samen mit Ruheperiode im Licht und Dunkel.

Folgende Samen wurden kurz nach der Ernte zum Keimen aus. gelegt. Der Verlauf ihrer Keimung ist aus der nachstehenden Tabelle ersichtiich.

1) Ich hebe ausdrücklich hervor, daß die Samen selbst gesammelt waren, da man bei $\mathrm{k}$ ä uflich e $\mathrm{n}$ Samen meist keine genauen Angaben über Provenienz und Erntedatum erhalten kann. Eine angenehme Ausnahme bildet die Firma F. Sünd ermann, Li n d a u i. B., die gutes Samenmaterial mit allen gewünschten Angaben liefert. 


\begin{tabular}{|c|c|c|c|c|c|c|c|c|c|c|c|c|c|c|c|}
\hline \multirow{2}{*}{$\frac{\text { Es keimen }}{\text { Nach Wochen }}$} & \multicolumn{8}{|c|}{ Im Licht } & \multicolumn{7}{|c|}{ Im Dunkel } \\
\hline & 1 & 2 & 3 & 5 & 16 & 20 & 21 & 23 & 1 & 2 & 4 & 6 & 16 & 20 & 23 \\
\hline Aethusa cyn. $\%$ & 8 & 8 & 2 & & 20 & 18 & & 10 & 6 & 6 & & & & & 4 \\
\hline Acer plat. & & 8 & 8 & & 12 & 8 & & 4 & & & 4 & 8 & 8 & 12 & \\
\hline Geran. pyr. & & 30 & 2 & & 2 & 2 & & 2 & 14 & 4 & & & & & 2 \\
\hline Ranunculusacer \% & & 2 & 2 & & 2 & 2 & & & & 4 & & & & & \\
\hline Oenothera $b . \quad \%$ & & 4 & & & & & & & 16 & 12 & & & & & \\
\hline Silene aucalis $\%$ & & 16 & & & & & & & 8 & & & & & & \\
\hline
\end{tabular}

Die Tabelle zeigt zunächst, daß die Keimung im Licht und Dunkel nicht gleich verläuft, wenn auch die Unterschiede nicht bedeutend sind. Es scheint der unausgeruhte Same gegen Lichteinflüsse viel empfindlicher zu sein als der ausgeruhte.

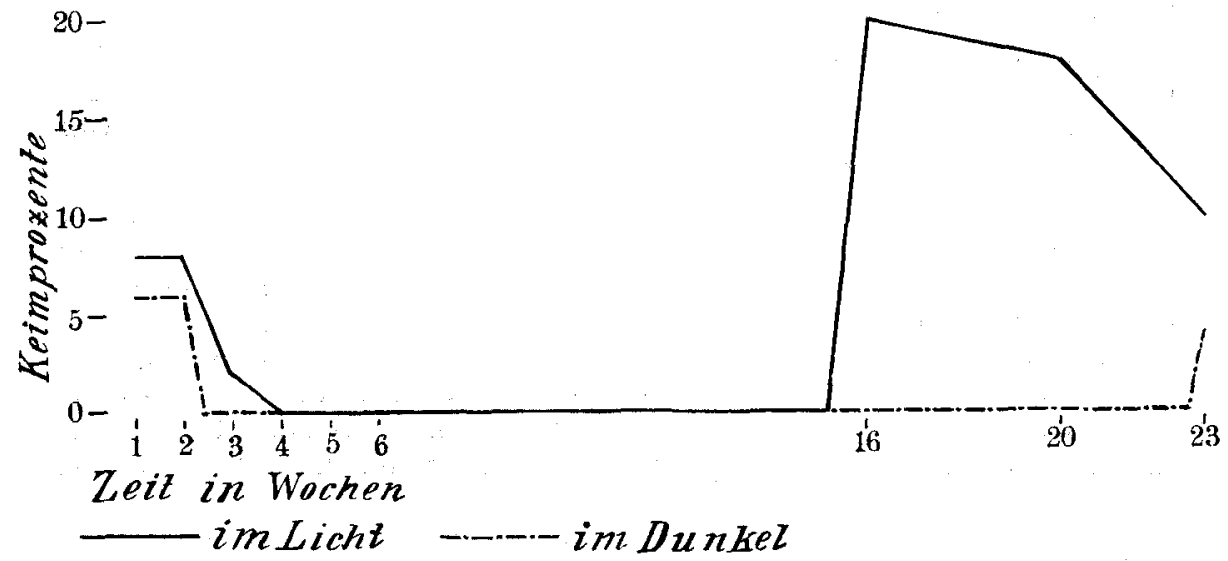

Abb. 1. Die Kurve zeigt dentlich, daß auch v or der Ruheperiode eine Keimung stattfindet.

Dieses Resultat dürfte für die jetzt modernen Lichtuntersuchungen wichtig sein, da man gewöhnlich mit nicht ausgeruhten Samen gearbeitet hat. Da bisher nur wenige Samen mit Ruheperiode bekannt waren, hat sich auch noch niemand die Frage vorgelegt, ob der wichtige Faktor Licht, bzw. Dunkel einen Einfluß habe, wenn man die Samen am Anfang und am Ende der Ruheperiode daraufhin untersucht.

Aus der Tabelle ist ferwer zu ersehen, daß die Samenrube keine allgemeine ist. Vielmehr keimt ein gewisser Prozentsatz in den ersten 
2-4 Wochen aus. Dann ruht der Samen 3-4 Monate und dann beginnt die Keimung aufs neue in kleinen Partien.

Es verhalten sich also keineswegs alle Samen keimungsphysiologisch gleich, sondern sie zeigen eine ziemlich ausgeprägte Individualität. Am anschaulichsten läßt sich der Verlauf der Ruheperiode durch eine Kurve darstellen, wie ich sie für Aethusa cynapium konstruiert habe. (Abb. 1.)

\section{Einfluß der Temperatur von $0^{\circ}$ a uf Samen mit Ruhe- periode.}

Damit hatte ich eine Reihe von Samen mit Ruheperiode gefunden und ging nun daran, zu untersuchen, ob diese durch Kälte abkürzbar sei. Ich verfuhr dabei analog wie in I.

Alle Versuche aber verliefen negativ. Die Temperatur von $0^{0}$ hat auf die Ruheperiode keinen Einflufs.

Ich setzte die Samen auch einmal dem strengen Frost aus, und da zeigte sich, daß die Keimung dadurch beschleunigt wurde, wie dies schon mehrere Forscher, allen voran Kinzel ${ }^{1}$ ), konstatierten.

Auf diese Verhältnisse ging ich natürlich nicht näher ein, da sie mich zu weit von meinem Thema abgeleitet hätten. Meine Absicht war ja bloß, den Einfluß der Temperatur von $0^{\circ}$ und um $0^{0}$ diesmal auch auf die Ruheperiode zu überprüfen. Es war dann noch ein zweiter Umstand maßgebend, der verlockenden Arbeit über den Einfluß dieser Temperaturen auf die Keimung der Samen mit Ruheperiode zu widerstehen. Das ist die Notwendigkeit der Versuchsunterbringung in Räumen mit konstanter Temperatur von $0^{0}$ bis $-12^{\circ}$. Solche Räume aber standen mir nicht zur Verfügung.

\section{Zur Keimung dimorpher Samen.}

Bei meinen Untersuchungen beobachtete ich einen Dimorphismus bei den Früchtchen mehrerer Tragopogon-Arten. (Siehe Abb. 2.) Beiderlei Früchtchen haben Riefen, welche dazwischen liegende Flächen begrenzen. Die rands tänd igen Früchtchen, welche dunkel, braun bis schwarz, gefärbt sind, zeigen sowohl auf den Riefen als auch auf den dazwischen liegenden Flächen stark entwickelte Höcker. Die scheibenständigen Frücht-

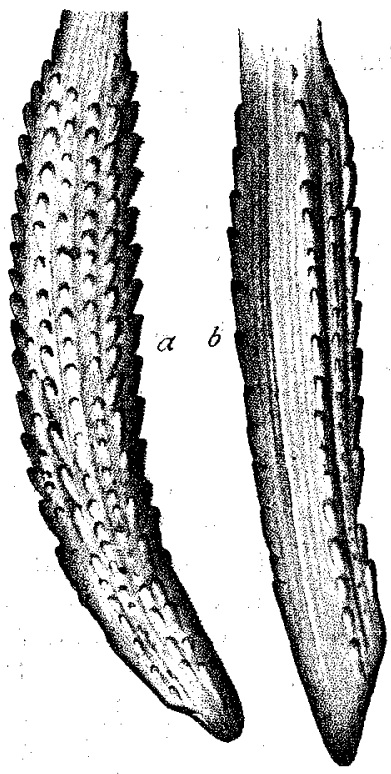

Abb. 2.

Dimorphe Früchtchen von Tragopogon dubius.

a) Randständig. Die Schale ist von großen, reihenweise angeordneten Höckern bedeckt. b) Scheibenständig.

Die Schale weist nur we nige Reihen schwach entwickelter Höcker auf. Vergr. ca. 8 mal. - Sohnabel und Pappus sind in der Zeichnung weggelassen.

1) 1. c. 
chen sind hell, gelblich, gefärbt und weisen nur auf den Riefen Höcker auf, die bedeutend schwächer entwickelt sind als bei den Randfrüchtchen. Die Flächen sind bei den Scheibenfrüchtchen von Höckern gänzlich frei. Diese Beschreibung bezieht sich nur auf extreme Fälle. Dazwischen finden sich, je nach der Stellung der Früchtehen, alle Übergänge. Die dazwischen liegenden Früchtchen zeigen Übergangs. formen. Festgestellt wurde dieser Dimorphismus bei ${ }^{1}$ ): Tragopogon dubius, orientalis und porrifolius. Von diesen Arten untersuchte ich T. dubius näher und stellte fest, dals der morphologischen auch eine keimungsphysiologische Verschiedenheit parallel steht. Nachfolgend eine Versuchsreihe, die dies veranschaulicht.

Die Früchtchen wurden ohne vorhergehende Quellung gleichzeitig ausgelegt. Es keimten die Früchtchen in nachstehenden Prozentzahlen nach Tagen:

\begin{tabular}{llrrrrrrr} 
& & 4 & 5 & 6 & 7 & 8 & 9 & 10 \\
Im Licht & Scheibenfrucht & 32 & 40 & 48 & 56 & 84 & 92 & 100 \\
\cline { 2 - 8 } Im Dunkel & Randfrucht & 0 & 32 & 44 & 68 & 96 & 96 & 100 \\
\cline { 2 - 8 } & Scheibenfrucht & 88 & 88 & 96 & 100 & & & \\
\cline { 2 - 9 } & Randfrucht & 72 & 92 & 96 & 100 & & &
\end{tabular}

Diese Zahlen zeigen, daß die Randfrucht zunächst schlechter keimt als die Scheibenfrucht, diese dann aber ein-, ja überholt. Ferner geht die Keimung im Dunkel schneller vor sich als im Licht.

Die Befunde schlieken sich also an jene von Becker, Baar u. a. $\left.a n^{2}\right)$.

\section{Zusammenfassung.}

Für die von mir untersuchten Samen konnten folgende Tatsachen festgestellt werden:

1. Folgenden Samen kommt eine Ruheperiode zu: Aethusa cynapium, Acer platanoides, Geranium pyrenaicum, Ranunculus acer, Oenothera biennis und Silene acaulis.

2. Weder auf die Keimung von Samen mit noch auf die Keimung von Samen oh $\mathrm{n}$ e Ruheperiode haben, unter meinen Versuchsbedingungen, bei den von mir untersuchten Arten, Schnee, Eis, Wasser und Luft von $0^{0} \mathrm{C}$ einen bemerkenswerten Eintlul.

3. Die Ruheperiode erstreckt sich nicht gleichmäßig auf alle Samen, sondern wird durch die Individualität der einzelnen Samen eingeschränkt, und zwar so, daß ein bestimmter Prozentsatz immer unmittelbar nach der Ernte auskeimt.

1) An dieser Stelle möchte ich Herrn Landesgerichtsrat C. A u s t meinen ergebensten Dank aussprechen, denn dadurch, daß er mir die Benützung seiner reichen Sammlungen erlaubte, war es mir möglich, diese interessanten Tatsachen festzustellen.

2, B e cker H. Über die Keimung verschiedenartiger Früchte und Samen bei derselben Spezies. Beih. z. Bot. Centrbl., Bd. XXIX, 1. Abt., 1913, p. $21 \mathrm{ff}$.

B a a $\mathrm{r}$ H, Zur Anatomie und Keimungsphysiologie heteromorpher Samen von Chenopodium album und Atriplex nitens. Sitzber. d. kaiserl, A kad. d. Wiss, in Wien, mathem.-naturw. Klasse, Bd. CXXII, Abt. I, Jänner 1913. 
4. Unausgerubte Samen sind im allgemeinen gegen Licht empfindlicher als ausgeruhte.

5. Die von mir untersuchten Samen versehiedener Provenienz und verschiedenen Erntedatums derselben Art zeigten keine Unterschiede in der Keimung.

6. Bei nachfolgenden Arten wurde ein Dimorphismus der Früchtchen festgestellt: Tragopogon dubius, T. orientalis und T. porrifolius.

7. Die dimorphen Früchtchen von Trugopogon dubius weisen auch ein keimungsphysiologisch verschiedenes Verhalten auf.

Am Schlusse meiner Arbeit ist es mir ein Bedürfnis, Herrn Prof. Dr. Hans Molisch für die Anregung zu dieser Arbeit und deren stete Unterstützung meinen tiefsten Dank auszusprechen. Degleichen danke ich herzlichst Herrn Prof. Dr. Oswald Richter für sein großes Interesse, das er meiner Arbeit entgegenbracht hat.

\section{Übersicht der aus der Bukowina bekannten Arten der Gattung Potentilla, L.}

Von Constantin Freíh. v. Hormuzaki (Czernowitz).

(Mit 6 Textabbildungen.)

(SchluB. I)

Spezieller Teil.

Genus Comarum L.

C. palustre L. Wie anderwärts nur auf Torfmooren, daher in der Bukowina auf die höhere montane Region beschränkt; Dornatal, 800 bis $900 \mathrm{~m}$ : Popeni bei Dorna-Candreni (leg. O. Freih. v. Petrino), DornaVatra. Die Exemplare dieser wenig variierenden Ast stimmen mit solchen aus Nordböhmen überein; die Blätter sind mit anliegenden kurzen Seidenhaaren spärlich behaart, auf der Unterseite etwas dichter, neigen also zur forma subsericea Becker.

Genus Potentilla L.

Sectio I. Potentillae trichocarpae.

Subsectio B. Nematostylae.

Grex 5. Palustres.

P. palustris Scop. = Comarum palustre L. (wie oben).

Grex 13. Fragariastrae.

P. alba L. Nur in der pontischen Region des Hügellandes; Onut am Dniester $(150-250 \mathrm{~m})$, leg. 0. Freih. v. Petrino; Prisacarein am linken Serethufer an südlichen Abhängen $(350-400 \mathrm{~m})$ auf Wiesen; die Exemplare sind stets breitblättrig, gehören also zur forma platyphylla Th. W.

1) Vgl. diese Zeitschr., 1914, p. 223-232. 